\section{GENOMIC DRIVERS OF LARGE B-CELL LYMPHOMA RESISTANCE TO CD19 CAR-T THERAPY}

${ }^{1}$ Michael Jain, ${ }^{2}$ Bachisio Ziccheddu, ${ }^{2}$ Caroline Coughlin*, ${ }^{1}$ Rawan Faramand, ${ }^{2}$ Anthony Griswold, ${ }^{1}$ Kayla Reid, ${ }^{2}$ Ola Landgren, ${ }^{1}$ Frederick Locke, ${ }^{2}$ Francesco Maura, ${ }^{1}$ Marco Davila, ${ }^{2} J o n a t h a n$ Schatz. 'Moffitt Cancer Center, Tampa, FL, United States; ${ }^{2}$ University of Miami Miller School of Medicine, Miami, FL, United States

Background CD19-directed chimeric antigen receptor-reprogrammed autologous $\mathrm{T}$ cells are breakthrough immunotherapies for heavily pretreated patients with diffuse large B-cell lymphoma (DLBCL), but across CAR-19 products, $\sim 60 \%$ of patients fail to respond or relapse. Inflammatory markers and clinical factors associate with impaired responses, but tumorintrinsic resistance drivers are largely undefined.

Methods To characterize the genomic mechanisms involved resistance to CAR-19, we interrogated whole genome sequencing (WGS) from 28 relapsed/refractory $(\mathrm{r} / \mathrm{r}$ ) aggressive lymphoma patients uniformly treated with axicabtagene ciloleucel (axi-cel).

Results Because prognostic factors defined in the frontline treatment setting are largely inapplicable to CAR-19, we leveraged the WGS data, including comparative analyses with untreated DLBCL cases in the Pan-Cancer Analysis of Whole Genomes (PCAWG) (figure 1). In analyses of individual mutated genes, TP53 was significantly enriched $(p=0.002)$ in CAR-19 patients, but did not predict outcome. However, mutations in either NFKBIA or MYC associated with worse PFS after CAR-19 $(p=0.04, p=0.025$ respectively). We next identified 12 single base substitution (SBS) mutational signatures in our cohort and found presence of APOBEC (SBS2 and SBS13) signatures associated with worse PFS, with $4 / 5$ patients progressing $(p=0.03)$. Copy number analysis by GISTIC2.0 revealed focal deletions of RHOA and RB1 to be significantly enriched in our cohort and independently predicted poor outcome $(\mathrm{p}=0.0007, \mathrm{p}=0.05$ respectively). WGS identifies structural variants and complex events. We found chromothripsis, a catastrophic shattering and reassembly of chromosomes, in $39.3 \%$ of $\mathrm{r} / \mathrm{r}$ DLBCL, which was strongly associated with poor CAR-19 outcome, with 9/11 affected cases progressing $(p=0.041)$. Finally, reduced expression $(n=3)$ or genomic alteration $(n=3)$ of CD19 did not associate with poor outcome. One case with durable response contained a sub-clonal CD19 mutation (L174V) previously reported as associated with CAR-19 resistance. These findings demonstrate predominance of CD19-independent resistance and indicate antigen-mediated tumor killing is not the only mechanism of tumor eradication. Genomic complexity appears to promote an immunosuppressive tumor microenvironment (TME), limiting CAR-19 efficacy.
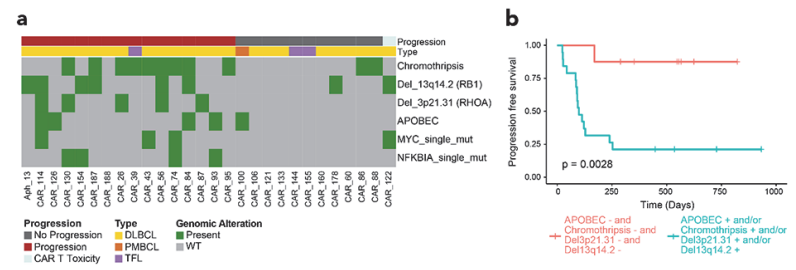

Abstract 675 Figure 1 Genomic alterations associated with disease progression. (a) The heatmap shows the significant genomic alteration present in at least 4 patients associated with progression after CD19 CAR-T cell therapy. (b) Kaplan-Meier curve of progression free survival with the combination of statistically significant genomic anomalies
Conclusions Leveraging the resolution of WGS, we observed that markers of genomic complexity (chromothripsis and APOBEC) and specific genomic alterations (RHOA and RB1 deletions) associate with resistance to CAR-19 immunotherapy for aggressive B-cell lymphomas (figure 1). 93.8\% of CAR-19 relapsed patients contained at least one or these genomic alterations. Recent patient data demonstrate that an immunosuppressed TME leads to CAR-19 failure. Combining these findings with our genomics findings, successful CAR-19 therapy must overcome the immune-exhausted TME to mobilize the host immune system and eliminate the tumor.

http://dx.doi.org/10.1136/jitc-2021-SITC2021.675 